\title{
Statistical properties of exoplanets
}

\section{The period-eccentricity relations of exoplanets and of binary stars}

\author{
J. L. Halbwachs ${ }^{1}$, M. Mayor ${ }^{2}$, and S. Udry ${ }^{2}$ \\ 1 Observatoire Astronomique de Strasbourg (UMR 7550), 11 rue de l'Université, 67000 Strasbourg, France \\ e-mail: halbwachs@astro.u-strasbg.fr \\ 2 Geneva Observatory, 51 chemin des Maillettes, 1290 Sauverny, Switzerland \\ e-mail: [Michel.Mayor;Stephane.Udry]@obs.unige.ch
}

Received 3 May 2004 / Accepted 19 October 2004

\begin{abstract}
A sample of spectroscopic binaries and a sample of single planetary systems, both having main-sequence solar-type primary components, are selected in order to compare their eccentricities. The positions of the objects in the $\left(P .\left(1-e^{2}\right)^{3 / 2}, e\right)$ plane is used to determine parts in the period-eccentricity diagram that are not affected by tidal circularization. The original eccentricities of binaries and planets are derived and compared. They seem to be weakly or not at all correlated with period in both samples, but two major differences are found:

(1) The tidal circularization of planetary orbits is almost complete for periods shorter than 5 days, but it is not visible when $P .\left(1-e^{2}\right)^{3 / 2}$ is longer than this limit. This suggests that the circularization occurs rapidly after the end of the migration process and is probably simultaneous with the end of the formation of the planet. By contrast, we confirm that the circularization of the binary orbits is a process still progressing a long time after the formation of the systems.

(2) Beyond the circularization limit, the eccentricities of the orbits of the planets are significantly smaller than those of binary orbits, and this discrepancy cannot be due to a selection effect. Moreover, the eccentricities of binaries with small mass ratios are quite similar to those of all binaries with $q<0.8$. This suggests that the low eccentricities of exoplanet orbits are not a consequence of low-mass secondaries in a universal process.

These remarks are in favor of the idea that binaries and exoplanets are two different classes of object from the point of view of their formation.
\end{abstract}

Key words. stars: binaries: general - stars: binaries: spectroscopic - stars: planetary systems stars: planetary systems: formation

\section{Introduction}

It is well known that the orbits of the exoplanets with periods larger than 5 or 6 days have eccentricities significantly larger than those of the giant planets of the solar system. Several mechanisms were proposed to explain this feature, but, up to now, none is fully convincing. It was proposed that eccentric orbits could be a consequence of the dynamic evolution of systems initially involving several planets (Rasio \& Ford 1996; Lin \& Ida 1997; Ford et al. 2001; Papaloizou \& Terquem 2001; Rice et al. 2003), but these models fail to produce the frequency of giant planets with semi-major axes smaller than about $1 \mathrm{AU}$. The giant planets close to their harboring stars are often assumed to be produced by migration within a disk (Ward 1997; Masset \& Papaloizou 2003, and references therein), but this process hardly produces eccentric orbits (Papaloizou et al. 2001; Thommes \& Lissauer 2003), although Goldreich \& Sari (2003) and Woolfson (2003) leave some room for hope. Therefore, it is tempting to consider that the exoplanets are generated by the same process as binary stars
(Stepinski \& Black 2000). This implies that giant exoplanets were not formed by gas accretion onto a heavy rocky core, as usually assumed, but by an alternative process. They could come from disk instabilities (Mayer et al. 2002; Boss 2002, 2003), but inward migration in a disk is then invoked again to explain the short-period orbits; alternatively, planets could be generated by fragmentation of a collapsing protostellar cloud, via filament condensation and capture (Oxley \& Woolfson 2004), or even exactly as stellar components in binary systems (see discussion in Bodenheimer et al. 2000, and references therein). However, these models may be efficient in forming massive planets or brown dwarfs, but not planets around 1 Jupiter mass or less.

Note that the binary formation models are not very satisfactory either (see the review by Tohline 2002). The large eccentricities of binaries are explained by fragmentation of collapsing cores and subsequent interactions between the forming stars (Bate et al. 2002; Goodwin et al. 2004), but, as for exoplanets, the simulations do not provide the high frequency 
of close systems. Moreover, statistical investigations on main sequence binaries (Halbwachs et al. 2003, Paper I hereafter) have shown that the close binaries (i.e. with semi-major axes less than a few AU) consist in two populations: one with large eccentricities and mass ratios less than 0.8 ("non-twins" hereafter), and one with moderate eccentricities and nearly identical components ("twins"). Additionally, the twins are more frequent among short-period binaries than among the others. At first, these properties were derived from binaries with F7-K primary components, but they are also valid for M-type dwarfs (Marchal et al. 2003).

In the present paper, the period-eccentricity diagram is used to compare the exoplanets with the binary stars: our main purpose is to investigate if the properties of exoplanets may be considered as an extrapolation of the properties of binaries in the range of very low mass ratios. This would indicate whether the formation processes of these objects are similar. In the course of the paper, a few other points are also treated: (1) the correlation between the eccentricity and the period or the angular momentum; (2) the relation between the eccentricity and the metallicity of planets; (3) the original distributions of eccentricities for planets and for binaries, considering the twins separately. Comparisons between planets and binaries in the period-eccentricity diagram were already presented by Mayor et al. (2001), Mazeh \& Zucker (2001), and Udry (2001), who concluded that planets and binaries are very similar when periods longer than 50 days are considered. However, their samples contained around 30 or 40 planets, and a many others have been discovered since. The question needs to be re-considered.

The interpretation of the period-eccentricity diagram is rather complex, and our investigations are based on the method presented in Sect. 2. Section 3 is dedicated to the binaries; we investigate if, additionally to the twins, other classes of mass ratio have specific distributions of eccentricities. A similar treatment is applied to exoplanets in Sect. 4. Binaries and exoplanets are compared in Sect. 5, in which we pay attention to the difference in the selection effects of both samples. The consequences of our results are discussed in Sect. 6.

\section{Method}

\subsection{Tidal effects}

We must pay attention to the fact that the periods $(P)$ and the eccentricities $(e)$ of the objects are modified by tidal interactions, especially when $P$ is short. As a consequence, the $(P-e)$ diagram may schematically be divided into two parts: the short periods, where the orbits are circular or have low eccentricities, and the periods longer than the circularization limit, hereafter called $P_{\text {cutoff }}$ (Mayor \& Mermilliod 1984; Duquennoy \& Mayor 1991). Several theoretical models were proposed to derived $P_{\text {cutoff }}$, and the treatment is not the same for binaries (Zahn 1992; Hut 1981, 1982; Keppens 1997, and references therein) and for planets (Goldreich \& Soter 1966; Trilling 2000). Moreover, several physical processes are invoked, each of them depending differently on the mass ratios of the systems.

Despite the complexity of the process, a few simple guidelines may be drawn. First of all, the efficiency of tides in modifying the orbits is very sensitive to the distance between the components. For a given system, the tidal torque depends on the orientation of the tidal bulge and on the separation between the components, $r$. It varies as $1 / r^{6}$ (Lecar et al. 1976). Therefore, for systems differing only by period, the transition from circularized orbits to orbits practically unaffected by tides is a narrow strip in the $(P-e)$ diagram (see the simulations by Witte $\&$ Savonije 2002). However, this does not mean that the systems with $P>P_{\text {cutoff }}$ may have any eccentricity. For a given period the systems with eccentric orbits have components much closer than the semi-major axis during a part of the period, and below a certain limit the orbit rapidly becomes circular. As a consequence, the upper part of the $(P-e)$ diagram is cleared even for $P>P_{\text {cutoff }}$. Note that the orbits that were originally eccentric do not keep the same period when they are evolving towards $e=0$ : when the primary star is a slow rotator, the orbit is circularized keeping the orbital angular momentum unchanged (Witte \& Savonije 2002; Hurley et al. 2002). Therefore, the semilatus rectum, $r_{\mathrm{sr}}=a\left(1-e^{2}\right)$, is conserved and it becomes the radius of the final circular orbit. As a consequence, the final period is:

$P_{\mathrm{sr}}=P .\left(1-e^{2}\right)^{3 / 2}$

where $P$ and $e$ refer to the original state of the system. This gives us a simple but efficient way to explore the transition from circularized orbits to orbits unaffected by tidal effects. In place of a $(P-e)$ diagram, the objects are plotted in the $\left(P_{\mathrm{sr}}-e\right)$ plane. Therefore, the evolution path toward a circular orbit is a vertical line in the diagram. Moreover, note that, for a wide range of eccentricities, the mean value of $1 / r^{6}$ for a complete orbit is approximately $1 / r_{\mathrm{sr}}{ }^{6}$ (Fig. 1). As a consequence, we expect that, when $r_{\mathrm{sr}}$ is small enough to permit efficient tidal effects for a given eccentricity, these effects will remain important during the evolution of the orbit, until they eventually lead to circularization. Therefore, the border between the circularized systems and the area nearly unaffected by tides in the $\left(P_{\mathrm{sr}}-e\right)$ diagram should appear very clearly. If the circularization were not at all related to the ages of the systems, the diagram should show a strong contrast, with the circular orbits on the left-hand side, and the orbits with any eccentricities, including the largest ones, immediately beyond the circularization limit. This is almost what is observed in reality, especially for the exoplanets (see Figs. 2 and 4 below).

In practice, however, a real sample is selected up to a maximum period $P_{\mathrm{Max}}$, and not all the eccentricities are permitted in the $\left(P_{\mathrm{sr}}-e\right)$ diagram. For a given $P_{\mathrm{sr}}$, the eccentricities range from 0 to the limit:

$e_{\text {Sup }}=\sqrt{1-\left(P_{\text {sr }} / P_{\text {Max }}\right)^{2 / 3}}$.

With $P_{\text {cutoff }}$ so determined, we derive from Eq. (1) the maximum eccentricity that the systems unaffected by tides in the $(P-e)$ diagram may have:

$e_{\text {Max }}=\sqrt{1-\left(P_{\text {cutoff }} / P\right)^{2 / 3}}$.

For comparing two samples which were have been differently affected by tidal circularization, it is necessary to restrict the comparison to the smallest of the two limits in $e_{\text {Max }}$ in order to find discrepancies coming only from the original distributions of $e$. 


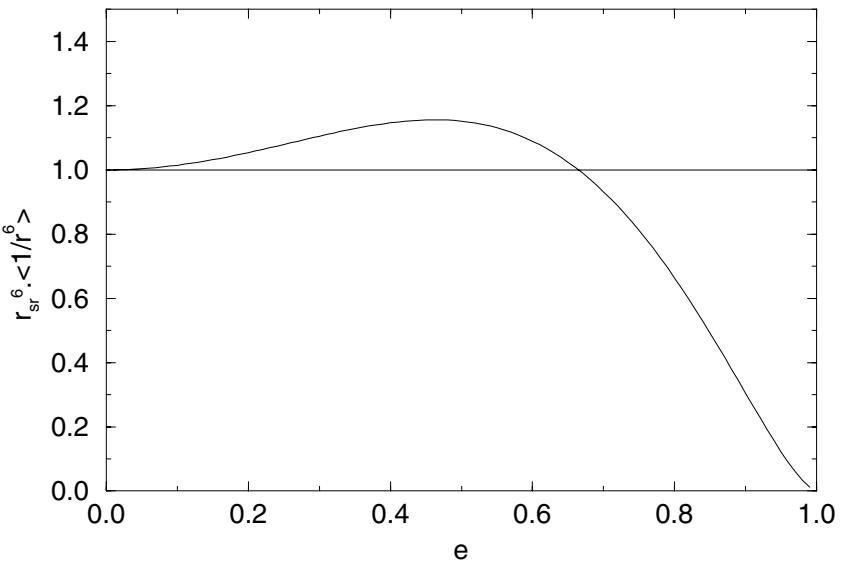

Fig. 1. The mean value of the $1 / r^{6}$ term of the tidal torque, compared to $1 / r_{\mathrm{sr}}^{6}$, where $r_{\mathrm{sr}}$ is the semilatus rectum. Except for very large eccentricities, $1 / r_{\mathrm{sr}}^{6}$ is an acceptable approximation of $\left\langle 1 / r^{6}\right\rangle$.

\subsection{Comparison of two samples}

The comparison between different samples of orbiting systems is based on the median eccentricities. Two approaches are used: the first is a visual examination, and the second is a statistical test. In the first approach, the median eccentricity of each sample is derived as a function of the period. In practice, for any period $P_{i}>P_{\text {cutoff }}$, the median eccentricity is derived from 6 systems taken among the periods closest to $P_{i}$. Except near the limit of the period range, 3 of these 6 systems have $P<P_{i}$ and the 3 others have $P>P_{i}$. The second approach is the statistical test used in Paper I: the two samples are merged, and the range of periods longer than $P_{\text {cutoff }}$ is divided into bins, each containing 12 systems, except for the last one which may contain up to 23 systems. The common median eccentricity is derived in each bin, and the systems below the median are counted for one of the two samples. If this sample actually belongs to the same statistical population as the other, the probability of getting any count, $P(k)$, obeys the hypergeometric distribution. The rejection threshold of the null hypothesis, $H_{0}$ : "all the systems are equivalent from the point of view of the eccentricities" is then derived. When the count $k$ is less than half the population of the considered sample, the rejection threshold of $H_{0}$ in a two-sided test is $2 \times P(i \leq k)$; on the contrary, it is $2 \times P(i \geq k)$ when $k$ is larger than the expected number.

Note that setting the content of the bins to 12 systems is a bit arbitrary. This number is neither too small to derive a reliable median nor too large to have a nearly constant period distribution within each bin. Using other numbers close to 12 would give other results, but it was verified that the differences are not important.

\section{The binaries}

\subsection{The binary sample}

The so-called extended sample of F7-K dwarf binaries selected in Paper I is used again. It consists of 89 spectroscopic binaries (SB) found in the solar neighborhood or in open clusters, with periods of up to 10 years. We already know that the

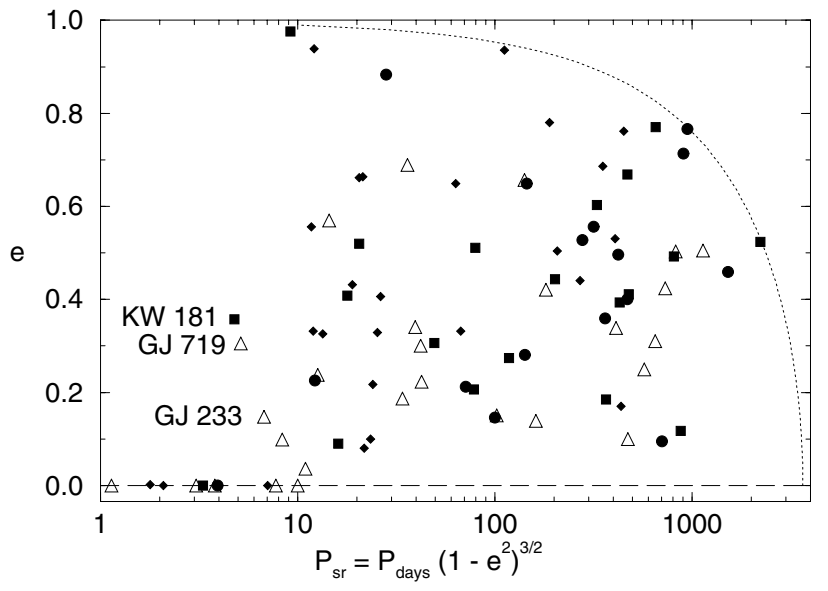

Fig. 2. Distribution of the $\mathrm{SB}$ in the $\left(P_{\mathrm{sr}}-e\right)$ diagram, where $P_{\mathrm{sr}}$ is the period that the SB would have if their orbits became circular with the same angular momenta. The symbols represent the systems with different mass ratios. The code is as follows: $q \leq 0.4$, circles; $0.4<$ $q \leq 0.8$, squares; $q \leq 0.8$ (maximum $q$ for SB 1 ) and minimum $q \leq 0.4$, small diamonds; $q>0.8$ (twins), open triangles. The thin dotted line is the limit $e_{\text {Sup }}$ corresponding to a maximum period of 10 years, as derived from Eq. (2).

twins have, on average, eccentricities smaller than the other binaries. However, before comparing binaries to exoplanets, it is worthwhile to see if the eccentricities of non-twin binaries depend on the mass ratios.

The mass ratios $q=\mathcal{M}_{2} / \mathcal{M}_{1}$ of the SB in the sample have been fixed for 58 binaries, thanks to the combination of the SB orbital elements with Hipparcos astrometric observations, or with photometric sequences in the open clusters (Paper I). For the other $31 \mathrm{SB}$, we derive intervals containing the actual mass ratios. The minimum limits are computed from the mass functions; the maxima are obtained differently for the nearby SB and for the cluster SB: the former all have $q<0.65$, since otherwise they would be double-lined SB with known $q$, and the latter have limits coming from their positions in the photometric sequence of the cluster.

In order to make visible a possible relation between the mass ratios and the eccentricities, the SB are distributed in several groups: $q \leq 0.40$ (16 SB), $0.40<q \leq 0.80$ (20 SB), and twins (27 SB); we still add a group containing all the SB with $q \leq 0.80$ (62 SB, including the 36 already in the first two groups).

\subsection{Limit of tidal circularization}

The SB are plotted in the $\left(P_{\mathrm{sr}}-e\right)$ diagram (Fig. 2), to delimit the part of the diagram affected by tidal circularization.

Several relevant features appear in Fig. 2. First of all, the range of eccentricities jumps from 0 to almost 1 between 4 and 9 days. All SB with $P_{\mathrm{sr}}$ shorter than 4 days are at present on circular orbits, and no evidence of circularization is visible when $P_{\text {sr }}$ exceeds 10 days. The SB with $P_{\text {sr }}$ between 4 and around 10 days have medium eccentricities, and some of them are even circularized. 
At least one of the short period SB may have been generated with a very large eccentricity, and may be too young for to have been circularized: KW 181 has the most eccentric orbit among the periods shorter than 10 days. This system belongs to the Praesepe cluster, and its age is therefore only $0.8 \times 10^{9}$ years. Its circularization will be complete within only $0.1 \times 10^{9}$ years (Duquennoy et al. 1992). The other two SB with $e>0.1$ and $P>10$ days may have eccentricities due to perturbations by a third component (Kozai mechanism or secular perturbations, see Mazeh \& Shaham 1979): GJ 719 has a CPM companion with a minimum separation of $280 \mathrm{AU}$ (Zuckerman et al. 1997), and GJ 233 is a visual binary with a possible period of 200 years (Heintz 1988). When these 3 SB are discarded, we find $P_{\text {cutoff }} \approx 8$ or 10 days, as in Duquennoy \& Mayor (1991).

Nevertheless, it is striking that all the SB with eccentric orbits and $P<10$ days have large mass ratios. In order to see if this feature is significant we look at the period-eccentricity diagram of the 205 SB found by Latham et al. (2002) among stars with large proper motions. They also found a range of period where circular orbits and eccentric orbits both exist, but between around 10 and 20 days (see their Fig. 9). It is visible in their plot that the double-lined SB (SB2), which have the largest $q$, are not abnormally frequent among the systems with eccentric orbits and short periods. Therefore, we admit that the frequency of twins with large eccentricity and $P<10$ days is just due to chance.

The sample of Latham et al. contains a few SB with circular orbits and periods between 10 and 20 days. However, they are stars with large proper motions, and they generally belong to the old galactic disk or even to the halo. Therefore, the long periods of some circularized orbits may be an effect of the ages of the systems, in agreement with theoretical predictions (see e.g. Duquennoy et al. 1992). Moreover, these old stars are not representative of the stars observed for planet detection. For the parent population of stars harboring planets, the limit of the area affected by tidal effects in the $\left(P_{\mathrm{sr}}-e\right)$ diagram is $P_{\text {cutoff }}=$ 10 days.

\subsection{The binaries in the $(P-e)$ diagram}

The $(P-e)$ diagram of the SB is plotted in Fig. 3. The values of the median of the four classes of $q$ are drawn in this figure for visual comparison.

As shown in Paper I, the twins often have below-average eccentricities when periods longer than 5 days are considered; the probability to get so large a discrepancy by chance, as derived by the two-sided test, is only $2.7 \%$. By contrast, the two groups with $q \leq 0.80$ have nearly the same distribution of eccentricities : the significance of the two-sided test is $100 \%$. In order to check that even the SB with the smallest mass ratios have the same eccentricity distribution as the others, the test is done again by comparing the SB with $q \leq 0.25$ (10 SB with $P>10$ days) to those with $0.25<q \leq 0.80$ (32 SB with $P>10$ days). Again, exactly half of the low-mass ratio SB are below the median, providing a $100 \%$ significance. Therefore, all the non-twin SB may be considered together in the comparison to the exoplanets.

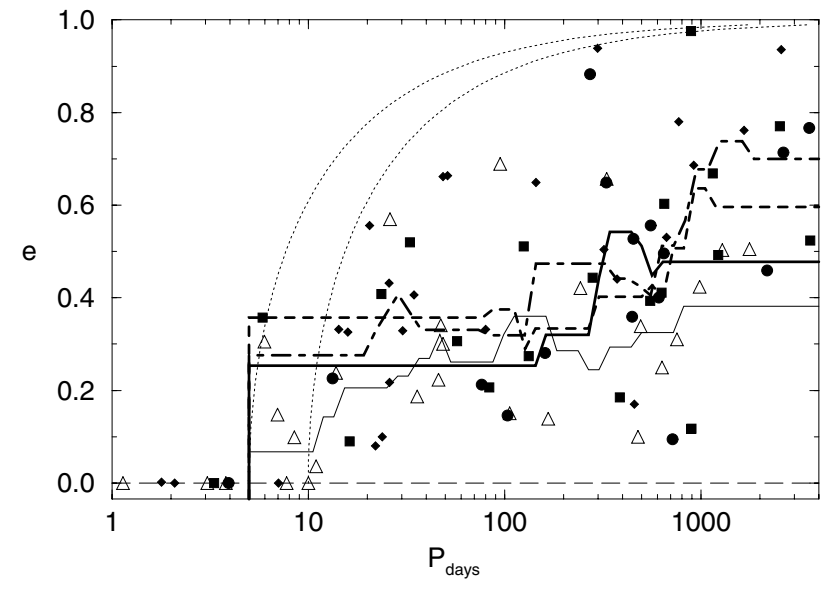

Fig. 3. Distribution of the SB in the period-eccentricity diagram. The symbols are the same as in Fig. 2. The lines refer to the median eccentricities of the different classes of mass ratios : $q \leq 0.4$, thick full line; $0.4<q \leq 0.8$, thick dashes; all $q \leq 0.8$, thick dot-dashed line; $q>0.8$ (twins), thin line. The 2 thin dotted lines are the limits of the location of SB with $P_{\text {sr }}$ between 5 and 10 days, as derived from Eq. (3).

We now come back to the distribution of $e$ of all SB. It is clearly visible in Fig. 3 that the median eccentricity increases with the period. However, we suspect that this may be entirely explained by tidal circularization, because the orbits having initially $P>P_{\text {cutoff }}$ and $e>e_{\text {Max }}$ are now circular with $P=P_{\text {sr }}<P_{\text {cutoff }}$. In other words, we want to see if the distribution of eccentricities depends on the periods, apart from the cut-off at $e=e_{\mathrm{Max}}$. A Spearman test is performed to check this hypothesis. In order to discard the area affected by tidal circularization, the test is restricted to the rectangular box $(P>20 \mathrm{~d}, e<0.61)$. The non-twins and the twins are considered separately. The Spearman correlation coefficient is 0.26 for the former, and 0.29 for the latter; taking into account the numbers of objects, the probabilities to get by chance values so far from zero are $10 \%$ and $20 \%$ respectively. Although these levels of significance are a bit low, they are still too large to reject the hypothesis that the eccentricities are not correlated with the periods as soon as the semilatus rectum of the orbit is larger than the radius of a circular orbit with period $P_{\text {cutoff }}$.

\subsection{Correlation eccentricity versus angular momentum}

Since $P_{\mathrm{sr}}$ is related to the angular momentum of the orbit, it seems relevant to see if the eccentricities are correlated with this parameter. This question looks similar to the $(P-e)$ correlation investigated just above, but it is different in reality, since changing the $(P-e)$ plane into the $\left(P_{\mathrm{sr}}-e\right)$ plane modifies the density in relation with the distribution of the periods. Therefore, the absence of correlation between $P$ and $e$ does not necessarily imply an absence of correlation between $P_{\text {sr }}$ and $e$, and vice versa.

A Spearman test is used again, but in a box in the $\left(P_{\mathrm{sr}}-\right.$ $e$ ) diagram. Since the selection of the sample was limited by the condition $P<10$ years, we must take into account the limit $e_{\text {Sup }}$ derived from Eq. (2) (the thin dotted line in Fig. 2). 
Therefore, the limits of the box considered in the Spearman test are $P_{\mathrm{sr}} \in[10 \mathrm{~d}, 789 \mathrm{~d}]$ and $e<0.8$, in order to avoid the area with $e>e_{\text {Sup }}$. Again, the rejection threshold of the hypothesis that $e$ and $P_{\text {sr }}$ are correlated is between 10 and 20\%, and the eccentricities may not depend on the angular momenta of the SB.

\section{The exoplanets}

\subsection{The planet sample}

We start from the up-to-date list of exoplanets that is provided on the Geneva web site ${ }^{1}$. However, several planets cannot be used in a comparison to the $\mathrm{SB}$, for several reasons:

- Since the binaries all have main-sequence primary components, the planets orbiting subgiant or giant stars are discarded. The specific problem of tidal circularization in a system containing an evolved star is thus avoided, as well as the uncertainty coming from the evaluation of the mass of an evolved primary component.

- The eccentricities must be reliable, and therefore derived from an orbit with good quality. For that purpose, the residuals of the radial velocity (RV) measurements, rms, are compared to the semi-amplitudes of the spectroscopic orbit, $K$. Five planets having rms larger than $K / 3$ are discarded. This criterion looks a bit rough, since several other factors could also be taken into account, such as the number of measurements, the phase distribution, and the fact that some orbits refer to a second companion. Nevertheless, it has the advantage of being simple, and it is worth noticing that all the SB in our sample satisfy this condition.

Moreover, the planets that were not followed by RV observations during a complete period are also discarded. Since this last condition results in removing the majority of the planets with periods longer than 2200 days, this value is adopted as a selection limit of the sample.

- It seems that the planets found in binary or multiple stellar systems have eccentricities smaller than the others when their periods are less than 30 days (Eggenberger et al. 2004). Three more planets are removed from the sample for this reason.

- Multiple planetary systems are supposed to be different from those with single planets, since the eccentricities may be affected by resonant perturbations. It is worth noticing that the distribution of these systems around the median eccentricity is not significantly different from that of the single planets: we find an excess of only one planet with a low eccentricity (when an excess of large $e$ is expected), and the probability to get this excess or a larger one just by chance is as large as $38 \%$. Nevertheless, for security, we still discard 14 planets belonging to multiple planetary systems.

A sample of 72 exoplanets orbiting main-sequence stars with periods shorter than 2200 days and with reliable orbits is thus finally obtained.

\footnotetext{
${ }^{1}$ http://obswww.unige.ch/Exoplanets
}

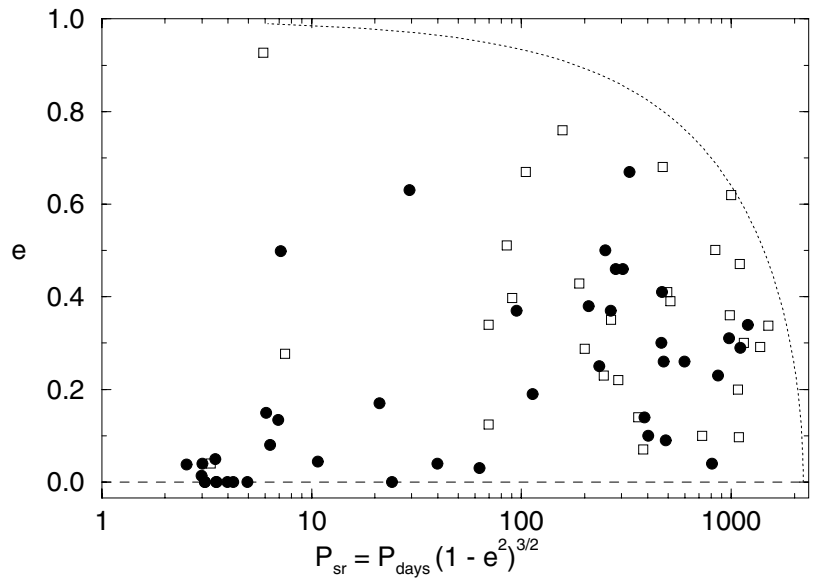

Fig. 4. Same as Fig. 2 for the planets. The circles are the planets with minimum mass $<2$ Jupiter, and the open squares refer to the others. The thin dotted line is the limit $e_{\text {Sup }}$ corresponding to a maximum period of 2200 days.

\subsection{The exoplanets in the $\left(P_{s r}-e\right)$ diagram}

As for the $\mathrm{SB}$, the planets are plotted on the $\left(P_{\mathrm{sr}}-e\right)$ diagram (Fig. 4), in order to investigate the effects of tidal circularization. The sample is split into two nearly equal groups, one containing the planets with minimum mass less than 2 Jupiter, and one with the planets heavier than this limit. In contrast to the SB, for which circular orbits and moderate eccentricities are mixed in a small range of $P_{\mathrm{sr}}$, the separation between the circularized orbits and the others is remarkably well determined, at $P_{\text {cutoff }}=5$ days. The most eccentric planetary orbit, HD $80606 \mathrm{~b}$ (Naef et al. 2001), is actually found for this period, but Wu \& Murray (2003) demonstrated that it may be excited by a distant companion through the Kozai mechanism.

It appears in Fig. 4 that the clustering of planets with $P<10$ days (Udry et al. 2003) is even more marked when $P_{\mathrm{sr}}$ is used in place of $P$ : the planets are concentrated in orbits with the semilatus recta corresponding to $P_{\mathrm{sr}}$ between 2.5 and 10 days, since we count 18 planets in this range, but only 1 between 10 and 20 days.

It would be relevant to check if the eccentricities are correlated with $P_{\mathrm{sr}}$, but our sample does not permit this: the detection of the planets is far from complete, and the incompleteness increases with the period. Therefore, since the eccentric orbits correspond to a longer period for a fixed $P_{\mathrm{sr}}$, the $\left(P_{\mathrm{sr}}-e\right)$ diagram of the planets is biased against large eccentricities. For that reason, the $\left(P_{\mathrm{sr}}-e\right)$ diagram of the planets can be used only for investigating the circularization limit. As a consequence, it cannot be used to compare the SB to the exoplanets in Sect. 5.

\subsection{The exoplanets in the $(P-e)$ diagram}

The $(P-e)$ diagram of the exoplanets is given in Fig. 5. Only 1 planet above 2 Jupiter masses has $P$ between 5 and 70 days. This paucity of heavy-mass planets with short periods has already been pointed out (Zucker \& Mazeh 2002; Udry et al. 2003), and it makes the median eccentricity of these planets unreliable in this range of period. A two-sided test based on 


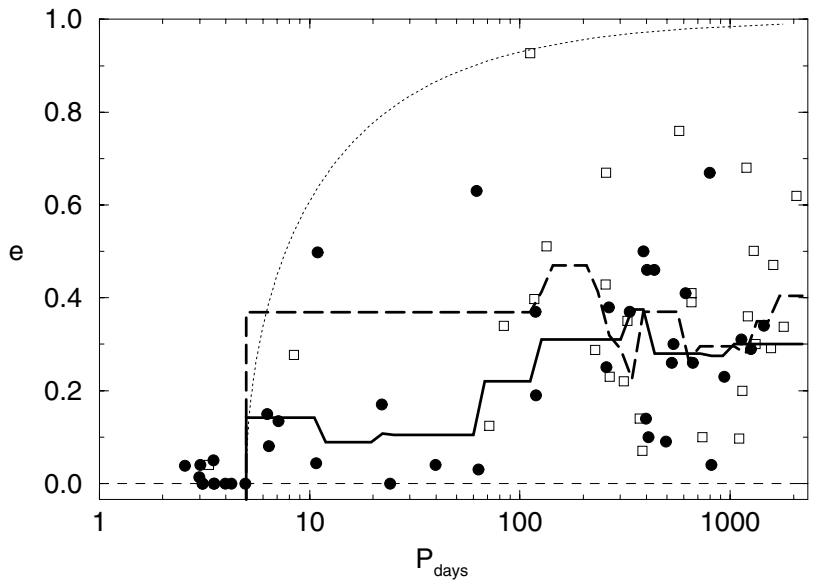

Fig. 5. Same as Fig. 3 for the exoplanets. The symbols are the same as in Fig. 4. The full line is the median eccentricity of the planets with minimum mass $<2$ Jupiter, and the dashes refer to the masses heavier than 2 Jupiter. The thin dotted line is the eccentricity $e_{\text {Max }}$ corresponding to a circularized period of 5 days.

the common median for $P$ in the range 5 to 2200 days shows that the probability to get differences at least as large as that obtained is $60 \%$. It is then quite possible that the eccentricities of planetary orbits are not related to the masses of the planets. This question is considered again in Sect. 5.2.

The median eccentricities of the exoplanets in Fig. 5 seem approximately constant. A test confirms this impression: the Spearman coefficient of the 52 planets with $P>20$ days and $e<0.78$ is 0.21 , providing a threshold between 10 and $20 \%$. It is thus not possible to rule out the hypothesis that, apart from circularization due to tidal effects, the distribution of the eccentricities of the planets is the same for any period between 20 and 2200 days. Therefore, if the eccentricities are modified by migration, they are changed almost independently of the periods. However, this applies essentially to periods longer than 200 days, since we have very few planets between 20 and 200 days.

\section{4. $(P-e)$ diagram and metallicities}

Santos et al. (2004) have shown that the percentage of stars harboring planets jumps from less than $5 \%$ to more than $20 \%$ when stars with $[\mathrm{Fe} / \mathrm{H}]$ larger than 0.2 are considered. In order to see if this limit of 0.2 dex also corresponds to other orbital properties, we use the $[\mathrm{Fe} / \mathrm{H}]$ of Santos et al. to distinguish the planets orbiting "metallic" stars and the others. In the $(P-e)$ diagram, we count 27 "metallic" planets, of which 14 have eccentricities smaller than the median. We conclude with a significance of $79 \%$ that metallicity is not related to the eccentricity, confirming the result obtained by Santos et al. (2003) with another test.

\section{The planets compared to the binaries}

\subsection{The period-eccentricity diagram}

Non-twin binaries and planets are plotted in the $(P-e)$ diagram in Fig. 6. The median eccentricities are derived from the

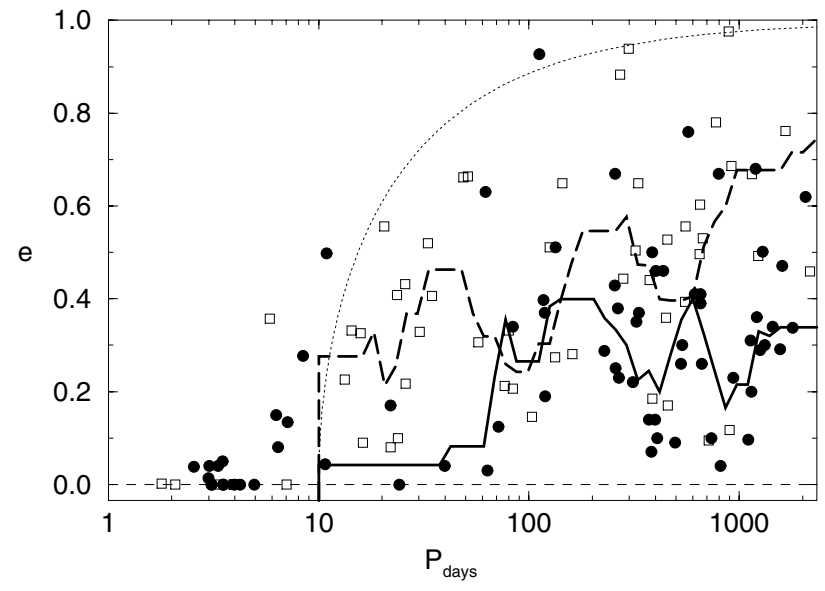

Fig. 6. The exoplanets compared to the non-twin binaries in the $(P-e)$ diagram. The circles refer to the planets, and the open squares to the SB with $q \leq 0.8$. The thin dotted line is $e_{\mathrm{Max}}$, the maximum eccentricity when the circularization limit is $P_{\text {cutoff }}=10$ days. The median eccentricities are derived after discarding the systems above this limit, in order to have the same selection effects for both samples. The median eccentricity of the planets is given by a full line, and that of the SB by a dashed line.

systems with $e<e_{\text {Max }}$. In order to make the comparison free of differences in the tidal circularization, $e_{\mathrm{Max}}$ is derived from Eq. (3) assuming $P_{\text {cutoff }}=10$ days for both samples. It appears clearly that, although all objects are distributed in the same area of the $(P-e)$ diagram, the planets have eccentricities that are on average smaller than those of the SB. The test of the distribution around the common median confirms this discrepancy: Among 53 planets included in a sample of 102 objects, we count 33 planets below the median eccentricity. The null hypothesis is rejected at the $1.7 \%$ level of significance.

It appears from Fig. 5 that several planets with long periods and minimum masses below 2 Jupiter have very small eccentricities. Therefore, although we have seen that the $(P-e)$ relation may be the same for all planets, it is relevant to compare the SB only to the planets with masses larger than 2 Jupiter. When the planets above 2 Jupiter masses are compared with all the non-twin SB, the null hypothesis is rejected again at the $4.8 \%$ level of significance, confirming that the heavy-mass planets have less eccentric orbits than the binaries.

\subsection{The intrinsic distribution of eccentricities}

A direct comparison of the distributions of the eccentricities of planets and of SB is not feasible, since the possible range of eccentricities varies with the period, and the distribution of $P$ is not the same for planets as for SB. Fortunately, another approach may be used to visually compare these objects, which is to derive the original distributions of eccentricities corrected for the bias coming from tidal circularization. The low significance values of the Spearman correlation tests performed above allows us to assume that, apart from the area affected by circularization, the eccentricity distribution does not vary with the period. Therefore, it is possible to derive the intrinsic distribution of the eccentricities for planets and for SB, using the 


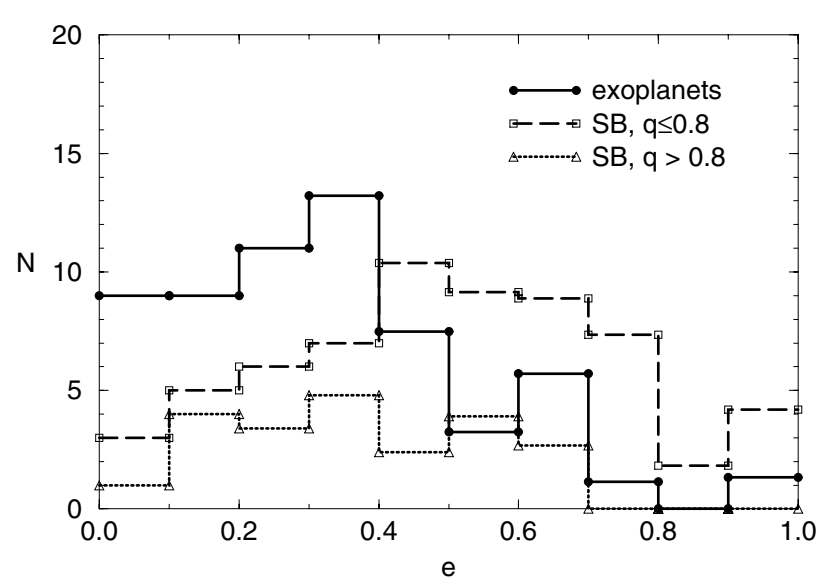

Fig. 7. Distribution of the eccentricities of SB and planets corrected for tidal circularization.

method of the "nested boxes". For that purpose, we use Eq. (3) to compute, for each system, the maximum eccentricity unaffected by tides, $e_{\text {Max }}$. We then apply the method given in the Appendix. The results are shown in Fig. 7.

The frequency of planets with low eccentricities is mainly due to the planets with masses below 2 Jupiter. The largest difference between the two groups of planets is obtained for $e=0.20$ exactly: $40 \%$ of the planets below 2 Jupiter masses have orbits with $e \leq 0.20$, instead of $18 \%$ for the others. However, a Smirnov test indicates that this discrepancy is far from sufficient to reject the hypothesis that all planets obey the same distribution, since the rejection threshold is as large as $38 \%$. An anonymous referee wondered whether this test could be affected by a bias related to the radial velocity semiamplitude. However, this bias is unfavorable to the planets with the lowest masses, since they are the most difficult to detect, and its efficiency is maximum when they have eccentric orbits (see Sect. 5.3 hereafter). As a consequence, correcting this bias would slightly decrease the proportion of $e \leq 0.2$ among the planets below 2 Jupiter, and then still increase the threshold of the test. We conclude again that we can assume that the eccentricity distribution of the planets does not depend on mass.

The differences pointed out by the tests and by the comparison of the medians are clearly visible in this figure. The maximum of the distribution is around 0.4 or 0.5 for the nontwin SB, and between 0.3 and 0.4 for the planets. Moreover, the distribution of $e$ decreases rapidly after the maximum for the planets, but it is rather flat until 0.8 for the non-twins SB. A comparison between the median $e$ of the exoplanets and of the twins could suggest that these two kinds of objects have similar distributions of eccentricities. However, differences appear in Fig. 7: instead of exhibiting a maximum like the exoplanets, the distribution of eccentricities of twins is nearly flat over a wide range, from $e=0.1$ to $e=0.7$.

\subsection{Is this difference real?}

We now want to check that the lack of planets with large eccentricities is not due to a selection effect against the detection of these systems. In contrast to the SB, the detection of planets is far from complete, and our sample is obviously biased in favor of those which are easiest to detect. The most obvious bias is against the detection of long period systems, but this does not affect the reliability of our test based on the median $e$; it just decreases the contribution of the long-period planets. However, another bias is directly related to the eccentricity: a large eccentricity increases the semi-amplitude in RV, but, at the same time, it decreases the rms of the RV measurements. Therefore, the detection of a system close to the limit of the instrument is more difficult when the eccentricity is large. Another consequence of this effect is a bias in the distribution of the periastron longitude, $\omega$. The orbits with $\omega$ around 0 or $\pi$ are more difficult to detect than those with $\omega$ around $\pi / 2$ or $3 \pi / 2$. This is visible, although not very significant, in our sample of planets: we count only 19 orbits with $\omega \in[-\pi / 4,+\pi / 4] \cup[3 \pi / 4,5 \pi / 4]$ among 43 planets with $e_{\operatorname{Max}}>e>0.1$ (the orbits with $e<0.1$ are not taken into account since $\omega$ is then not reliable).

Simulations have been performed to investigate if this bias may explain the discrepancy between planets and SB. Each planet receives the eccentricity of a SB, randomly taken among the $10 \mathrm{SB}$ with periods closest to that of the planet. The periastron longitude of the planet is randomly generated, and 3 radial velocity measurements are produced for 3 epochs randomly chosen, adding errors drawn from the residual rms of the true orbit; (in reality, each star observed for planet detection receives much more than 3 observations, but our aim is to derive an upper limit to the bias). When the standard deviation of the simulated RV is larger than the threshold corresponding to $P\left(\chi^{2}\right)=1 \%$, the planet is counted as detected; if not, another eccentricity is generated, and the simulation of the detection is performed again, until the planet satisfies the detection condition. When the complete sample has thus been detected by the simulation, the test of the median eccentricity in the $(P-e)$ diagram is performed. The simulation of the diagram is repeated 50000 times.

It appears from this calculation that the effect of the bias is to shift on average 0.6 more planets below the common median. Assuming that the number of planets below the median would be 32 in the absence of bias (instead of 33, see Sect. 5.1), the rejection threshold of $H_{0}$ becomes $4.7 \%$. This is still small enough to maintain rejection. At the same time, we count the planets with $\omega \in[-\pi / 4,+\pi / 4] \cup[3 \pi / 4,5 \pi / 4]$ which are detected in the simulation. Among the planets with $e>0.1$, their proportion is $45.5 \%$, in very nice agreement with the observed one, which is $19 / 43=44 \%$. We conclude then that the bias against detection of orbits with large eccentricities cannot explain the excess of planets with $e$ smaller than the common median in the $(P-e)$ diagram.

\section{Discussion and conclusion}

We have found some relevant features in comparing the eccentricities of the SB to those of the exoplanets :

- The $\left(P_{\mathrm{sr}}-e\right)$ diagram, based on $P_{\mathrm{sr}}$ defined in Eq. (1), is a powerful tool for determining the limit between the circularized orbits and the others. The contrast between the two areas in the diagram suggests that the tidal effects are 
efficient only when the semilatus rectum of the orbit is less than a fixed limit.

- The transition from the circularization to the part not affected by tidal effects looks sharper for the exoplanets than for the SB. For the SB, it corresponds to $P_{\text {sr }}$ between around 5 and 10 days, in agreement with Mathieu \& Mazeh (1988), Duquennoy et al. (1992), Mathieu et al. (1992) and Witte \& Savonije (2002), who consider that circularization is not restricted to the time of binary formation, but is still progressing during the whole lifetime of the main sequence components. For the exoplanets, the fast transition observed for planets with different ages is consistent with the idea that the tides were efficient only during the formation of the system. The inefficiency of tides for a formed planet is in agreement with the circularization time derived by Zahn (1977), which is a function of $(1+q) / q$. If the planets were brought closer to their host stars by migration, that means that migration occurred when the formation of the planets or of the host stars was not completed. Tidal circularization was then dominated by the tidal bulge on the planet, which was hotter, and therefore larger than it is today.

- Before tidal effects had modified them, the eccentricities of planets or binaries were not strongly related to the periods, or to the angular momenta. It is even quite possible that they were not correlated at all with these parameters, since the absence of correlation is not clearly rejected by statistical tests, and also because our assumption that tides did not affect at all the orbits with $P_{\mathrm{sr}}>P_{\text {cutoff is possibly a bit }}$ too simple. Since the planets in the sample are supposed to have migrated, this suggests that migration did not alter the eccentricities significantly; alternatively, it is possible that the eccentricities were modified, but almost independently of the final periods.

- The exoplanets have orbits with eccentricities significantly smaller than those of the SB with the same period and with mass ratios larger than 0.8 (the non-twin binaries). A similar feature has already been pointed out for $P<50$ days (Udry 2001), but neglecting the difference between the distributions of periods of binaries and of planets. Moreover, it is now certain that the low eccentricities of planets are not an effect of the selection of the observed sample. Additionally, it seems that the distributions of the eccentricities are not related to the masses of the companions, neither among the non-twin binaries, nor among the planets. Therefore, this discrepancy is probably not an effect of the low masses of planets in a formation/evolution process common to planets and binaries: this would imply a process depending on the secondary mass, but only around the transition between stellar and planetary companions. In fact, the SB most similar to the planets are the twins, perhaps because these systems were also interacting with a disk at the time of their formation, as Tokovinin (2000) suggested.

Our most relevant conclusion is that the eccentricities of the exoplanet orbits are rather in favor of the hypothesis that exoplanets and binary stars are not the products of the same physical process. After the "brown dwarf desert" (Halbwachs et al. 2000) separating the stellar components from the planets in the distribution of the secondary masses, it is another argument in that sense which was derived from statistical investigations.

Acknowledgements. We are grateful to Piet Hut and Jean-Paul Zahn for their explanations and for their valuable comments on the draft version of the paper. Douglas Heggie carefully read a preliminary version and added relevant corrections. An anonymous referee made valuable comments. The A\&A language editor, Jet Katgert, corrected the English. The selection of the exoplanets was partly based on data taken from Simbad, the database of the Centre de Données astronomiques de Strasbourg.

\section{Appendix A: The method of the nested boxes applied to the distribution of eccentricities}

This method was initially dedicated to the derivation of a biasfree distribution of mass ratios of visual binaries (Halbwachs 1983; Halbwachs et al. 1997), as is explained in detail in Halbwachs (2001). It is adapted hereafter to the derivation of the intrinsic distribution of the eccentricities.

We consider a sample with periods $P$ larger than $P_{\text {cutoff }}$, the period corresponding to tidal circularization, as explained in Sect. 2.1. For each system, $P$ and $P_{\text {cutoff }}$ are used to derive $e_{\mathrm{Max}}$, the maximum eccentricity of the orbits unaffected by tidal effects. The systems having eccentricities $e>e_{\text {Max }}$, if any, are discarded from the sample.

The principle of method is as follows:

- We define a first "box" by setting a small minimum value of $e_{\text {Max }}$, called $e_{1}$ hereafter. The eccentricities of the systems having $e_{\operatorname{Max}} \geq e_{1}$ are used to derive a first estimation of the intrinsic distribution of eccentricities, $f_{1}(e)$, which is defined from $e=0$ up to $e=e_{1}$. Since $e_{1}$ is small, this distribution is based on a large number of systems, and it is fairly reliable; unfortunately, it concerns only a small range of eccentricities.

- A second box is defined, using a limit $e_{2}>e_{1}$. The eccentricities of the systems with $e_{\mathrm{Max}} \geq e_{2}$ provide a second estimation of the distribution, $f_{2}(e)$, which applies to $e \leq e_{2}$. We have then an estimation of $f(e)$ which is valid between $e_{1}$ and $e_{2}$, but for $e \leq e_{1}, f_{2}(e)$ is less reliable than $f_{1}(e)$, since it is derived from fewer systems in this range.

- The two boxes defined above are "nested", since the systems with $e \leq e_{1}$ belonging to the second box are all also present in the first box. This common part is used to derive $f(e)$ by connecting $f_{2}\left(e>e_{1}\right)$ to $f_{1}\left(e \leq e_{1}\right)$. Let $N_{1}$ and $N_{2}$ be the numbers of systems in the first box and in the second box, respectively. If the second box contains $n_{2}\left(e_{1}\right)$ systems with $e \leq e_{1}$, the best estimation of $f(e)$ is:

$f_{1,2}(e)=f_{1}(e)+\theta\left(e-e_{1}\right) \frac{N_{1}}{n_{2}\left(e_{1}\right)} f_{2}(e)$

where $\theta$ is the Heaviside function; it is unnecessary to normalized the distributions so early in the calculation.

- We may still add several boxes, using limits $e_{i}$ with increasing values. If $N_{i-1}$ is the norm of the distribution derived 
from the boxes 1 to $i-1, f_{1, i-1}$, and if box $i$ contains $n_{i}\left(e_{i-1}\right)$ systems with $e \leq e_{i-1}$, Eq. (A.1) becomes :

$$
f_{1, i}(e)=f_{1, i-1}(e)+\theta\left(e-e_{i-1}\right) \frac{N_{i-1}}{n_{i}\left(e_{i-1}\right)} f_{i}(e) .
$$

The distribution is normalized after adding the last box.

In practice, the values of the $e_{i}$ terms are $i \times 0.01$ when the eccentricities are provided with two decimals, in order to take into account all the systems with $e \leq e_{\mathrm{Max}}$. At the end of the calculation, the 0.01-bins are merged into 0.1-bins, in order to make the final distribution more readable.

\section{References}

Bate, M. R., Bonnell, I. A., \& Bromm, V. 2002, MNRAS, 336, 705 Bodenheimer, P., Hubickyj, O., \& Lissauer, J. J. 2000, Icarus, 143, 2 Boss, A. P. 2002, ApJ, 576, 462

Boss, A. P. 2003, ApJ, 599, 577

de Pater, I., \& Lissauer, J. 2001, Planetary Sciences (Cambridge: Cambridge University Press)

Duquennoy, A., \& Mayor, M. 1991, A\&A, 248, 485

Duquennoy, A., Mayor, M., \& Mermilliod, J. C. 1992, in Binaries as Tracers of Stellar Formation, ed. A. Duquennoy, \& M. Mayor (Cambridge: Cambridge Univ. Press), 52

Eggenberger, A., Udry, S., \& Mayor, M. 2004, A\&A, 417, 353

Ford, E. B., Havlickova, M., \& Rasio, F. A. 2001, Icarus, 150, 303

Goldreich, P., \& Sari, R. 2003, ApJ, 585, 1024

Goldreich, P., \& Soter, S. 1966, Icarus, 5, 375

Goodwin, S. P., Whitworth, A. P., \& Ward-Thompson, D. 2004, A\&A, 414,633

Halbwachs, J. L. 1983, A\&A, 128, 399

Halbwachs, J. L. 2001, in Etoiles doubles, des étoiles à grandes séparations aux binaires X, ed. D. Egret, J. L. Halbwachs, \& J. M. Hameury, 35, http://astro.u-strasbg.fr/goutelas/g2000/

Halbwachs, J.L., Piquard, S., Virelizier, P., et al. 1997, ESA SP-402, 263

Halbwachs, J. L., Arenou, F., Mayor, M., Udry, S., \& Queloz, D. 2000, A\&A, 355, 581

Halbwachs, J. L., Mayor, M., Udry, S., \& Arenou F. 2003, A\&A, 397, 159 (Paper I)

Heintz, W. D. 1988, AJ, 96, 1072

Hurley, J. R., Tout, C. A., \& Pols, O. R. 2002, MNRAS, 329, 897

Hut, P. 1981, A\&A, 99, 126

Hut, P. 1982, A\&A, 110, 37

Keppens, R. 1997, A\&A, 318, 275

Latham, D. W., Stefanik, R. P., Torres, G., et al. 2002, AJ, 124, 1144

Lecar, M., Wheeler, J. C., \& McKee, C. F. 1976, ApJ, 205, 556
Lin, D. N. C., \& Ida, S. 1997, ApJ, 477, 781

Marchal, L., Delfosse, X., Forveille, T., et al. 2003, in Brown Dwarfs, ed. E. Martin (Astron. Soc. Pacific), IAU Symp., 211, 311

Masset, F. S., \& Papaloizou, J. C. B. 2003, ApJ, 588, 494

Mathieu, R. D., \& Mazeh, T. 1988, ApJ, 326, 256

Mathieu, R. D., Duquennoy, A., Latham, D., et al. 1992, in Binaries as Tracers of Stellar Formation, ed. A. Duquennoy, \& M. Mayor (Cambridge: Cambridge Univ. Press), 278

Mayer, L., Quinn, T., Wadsley, J., \& Stadel, J. 2002, Science, 298, 1756

Mayor, M., \& Mermilliod, J. C. 1984, in Observational Tests of the Stellar Evolution Theory, ed. A. Maeder \& A. Renzini (Reidel Publ. Comp.), IAU Symp., 105, 411

Mayor, M., Udry, S., Halbwachs, J. L., \& Arenou, F. 2001, in The Formation of Binary Stars, ed. H. Zinnecker \& R.D. Mathieu, IAU Symp., 200, 45

Mazeh, T., \& Shaham, J. 1979, A\&A, 77, 145

Mazeh, T., \& Zucker, S. 2001, in The Formation of Binary Stars, ed. H. Zinnecker, \& R.D. Mathieu, IAU Symp., 200, 519

Naef, D., Latham, D. W., Mayor, M., et al. 2001, A\&A, 375, L27

Oxley, S., \& Woolfson, M. M. 2004, MNRAS, 348, 1135

Papaloizou, J. C. B., Nelson, R. P., \& Masset, F. 2001, A\&A, 366, 263

Papaloizou, J. C. B., \& Terquem, C. 2001, MNRAS, 325, 221

Rasio, F. A., \& Ford, E. B. 1996, Science, 274, 954

Rice, W. K. M., Armitage, P. J., Bonnell, I. A., et al. 2003, MNRAS, 346, L36

Santos, N. C., Israelian, G., Mayor, M., Rebolo, R., \& Udry, S. 2003, A\&A, 398, 363

Santos, N. C., Israelian, G., \& Mayor, M. 2004, A\&A, 415, 1153

Stepinski T. F., \& Black D. C. 2000, A\&A, 356, 903

Thommes, E. W., \& Lissauer, J. J. 2003, ApJ, 597, 566

Tohline, J. E. 2002, ARA\&A, 40, 349

Tokovinin, A. A. 2000, A\&A, 360, 997

Trilling, D. E. 2000, ApJ, 537, L61

Udry, S. 2001, in Étoiles doubles, des étoiles à grandes séparations aux binaires X, ed. D. Egret, J. L. Halbwachs, \& J. M. Hameury, 141, http://astro.u-strasbg.fr/goutelas/g2000/

Udry, S., Mayor, M., \& Santos, N. C. 2003, A\&A, 407, 369

Ward, W. R. 1997, Icarus, 126, 261

Witte, M. G., \& Savonije, G. J. 2002, A\&A, 386, 222

Woolfson, M. M. 2003, MNRAS, 340, 43

Wu, Y., \& Murray, N. 2003, ApJ, 589, 605

Zahn, J. P. 1977, A\&A, 57, 383

Zahn, J. P. 1992, in Binaries as Tracers of Stellar Formation, ed. A. Duquennoy, \& M. Mayor (Cambridge: Cambridge Univ. Press), 253

Zucker, S., \& Mazeh, T. 2002, ApJ, 568, L113

Zuckerman, B., Webb, R. A., Becklin, E. E., McLean, I. S., \& Malkan, M. A. 1997, AJ, 114, 805 